\title{
Validation and assessment of an accurate approach to the correlation problem in density functional theory: The Krieger- Chen-Iafrate-Savin model
}

Julien TOULOUSE ${ }^{\mathrm{a}}$, Andreas $\mathrm{SAVIN}^{\mathrm{b}}$ and Carlo ADAMO $^{\mathrm{a}, *}$

a) Laboratoire d'Electrochimie et de Chimie Analytique, UMR 7575, ENSCP, 11 rue P. et M. Curie, F-75231 Paris Cedex 05, France.

b) Laboratoire de Chimie Théorique, UMR 7616, Université P. et M. Curie, 4 place Jussieu, F-75252 Paris Cedex 05, France.

July 24, 2002

\begin{abstract}
In the present paper, we validate and assess a meta-GGA correlation functional (KCIS), whose form and parameters are entirely derived only from first-principles criteria. In particular, we have carried out a detailed comparison with the most common, parameterized correlation functionals. Next, we propose a new model in which the KCIS correlation is integrated in a hybrid Hartree-Fock/Density Functional Theory scheme. In such approach only one, or two in the G2-optimized version, parameters are adjusted on experimental data, all the others being derived from purely theoretical considerations. The results obtained for a set of molecular properties, including $\mathrm{H}$-bonded complexes, proton transfer model, $\mathrm{S}_{N} 2$ reaction and magnetic (EPR) properties, are satisfactory and comparable, if not better, with those delivered by the most common functionals including heavy parameterization. The way in which the whole functional is derived and the few empirical parameters used make the new exchange-correlation functional widely applicable.
\end{abstract}

* Corresponding author: adamo@ext.jussieu.fr 


\section{Introduction}

Density Functional Theory (DFT), combining good performances and low computational costs, is nowadays one of the most powerful and reliable tools of quantum chemistry for the computation of the electronic structure of atoms, molecules or solids [1]. In the application of this theory within the Kohn-Sham formalism, the total electronic energy can be exactly expressed as a functional of the electron density, the only contribution that needs to be approximated being the exchange-correlation term [2]. Finding improved approximations to this contribution is therefore of vital importance in chemical applications to obtain accurate numerical molecular properties $[3,4]$.

A large number of approximations have been developed, ranging from the simple local density approximation (LDA) involving only the electron density $\rho$ to the generalized gradient approximations (GGA's) where the gradient of the density $\nabla \rho$ has been introduced to model the inhomogeneous character of molecular and real chemical systems. More recently, a new generation of functionals, called meta-GGA's and including the laplacian of the density $\left(\nabla^{2} \rho\right)$ and/or the kinetic energy density $(\tau)$, have attracted increasing attention. Introducing more semi-local or non-local information compared to previous approximations, these meta-GGA functionals are very promising but have not been fully explored yet [5].

Two extreme strategies can be defined in designing new approximations. The first approach consists in proposing an empirical formula containing some parameters fitted on experimental data, with the related danger of obtaining functionals adapted only to the applications for which they have been developed. The second way is represented by designing a mathematical form which satisfies some (eventually the largest possible number of) rigorous theoretical conditions of the exact exchange-correlation functional. This approach does not assure the "chemical" applicability of the developed functional. Of course the two philosophies are compatible and can be mixed, as recently showed [6,7].

In practice, any functional is arbitrarily separated into an exchange and a correlation contributions, and approximated forms are often developed in an independent way. For instance, a large number of approximations have been proposed for the exchange term representing the major part of the combined contribution to the total electronic energy (see for instance reference 8-12). In contrast, relatively few forms have been proposed for correlation functionals, perhaps due to the greater difficulty to represent this contribution. Here we recall some of the most common correlation forms, like those developed by Perdew and co-workers 
(like the P86, PW91 or PBE) [13-15], which are based on a GGA philosophy, while, only more recently, meta-GGA correlations appeared [16-19]

Aside from these expressions, all rooted on the free-electron gas, several functionals based on the Colle-Salvetti approach [20] have been proposed, where the inhomogeneity of the systems is introduced via the second-order reduced density matrix [21-23]. The most known derivation has been developed by Lee, Yang and Parr and it is one of the most common and successful correlation functionals (usually referred to as LYP) [21].

In this context, Krieger, Chen, Iafrate and Savin have recently proposed a new meta-GGA approach (hereafter referred to as KCIS), based on the idea of a uniform electron gas with a gap in the excitation spectrum [24,25]. The KCIS functional is, in our opinion, particularly appealing since it is built only from physical considerations, it preserves many of the know properties of the exact correlation energy (such as the so-called PBE conditions [15]) and it has no empirical parameters. Despite its promising features, the KCIS functional has never been tested beyond atomic or small molecular systems [26].

The aim of this paper is, therefore, to explore the limits of the applicability of such a functional to chemical systems and to compare the KCIS performances with those provided by the most common correlation functionals. To this end, several exchange functional have been chosen as counter part either in pure DFT approaches or in a hybrid HF/DFT schemes [27]. As in our previous works [7,10], we have tested the exchange-correlation functionals obtained in these ways by computing properties concerning a number of chemical systems or processes involving both covalent and weak interactions.

\section{Exchange and Correlation Functionals used.}

The KCIS correlation functional has the following expression [5]:

$$
E_{c}\left[\rho_{\alpha}, \rho_{\beta}\right]=\int F\left(\rho_{\alpha}, \rho_{\beta}, \nabla \rho_{\alpha}, \nabla \rho_{\beta}, \tau_{\alpha}, \tau_{\beta}\right) d \mathbf{r}
$$

where

$$
F=\rho \varepsilon_{c}^{G G A G A P}\left(\rho_{\alpha}, \rho_{\beta}, \nabla \rho\right)-\sum_{\sigma=\alpha, \beta} \frac{\tau_{\sigma}^{W}}{\tau_{\sigma}} \rho_{\sigma} \varepsilon_{c}^{G G A G A P}\left(\rho_{\sigma}, 0, \nabla \rho_{\sigma}\right)
$$

In equation (2), $\varepsilon_{c}^{G G A G A P}$ is the correlation energy per particle of a uniform electron gas with a gap in the excitation spectrum and including gradient corrections, and $\tau_{\sigma}^{W}$ is the Weizsäcker kinetic energy density:

$$
\tau_{\sigma}^{W}(\mathbf{r})=\frac{1}{8} \frac{\left|\nabla \rho_{\sigma}(\mathbf{r})\right|^{2}}{\rho_{\sigma}(\mathbf{r})}
$$


Correlation functionals usually transfer the correlation energy from the uniform electron gas to the real system, eventually adding some gradient corrections. However, these two systems are very different: the spectrum of the uniform electron gas is a continuum of states which are occupied up to the Fermi level and unoccupied above. On the contrary, the molecular or atomic spectrum is made of discrete states which are occupied up to the HOMO orbital and unoccupied up to the ionization threshold, followed by a continuum of unoccupied states. It is therefore more judicious to model the correlation energy by starting from a uniform electron gas with a gap in the excitation spectrum roughly corresponding to the difference between the HOMO energy and the ionization threshold of the real system. It has been chosen to express this gap as the simplest semi-local functional positive everywhere and reducing to the ionization potential in the large $\mathbf{r}$ limit:

$$
G[\rho]=\frac{1}{8} \frac{|\nabla \rho|^{2}}{\rho^{2}}
$$

Furthermore, $\varepsilon_{c}^{G G A G A P}$ has been built so as to the three PBE theoretical conditions are fulfilled by the KCIS functional. Besides, the second term in expression (2) ensures the selfinteraction correction (SIC) of the functional which enables the correlation energy to vanish in the case of one-electron systems [28].

Since, any form for exchange functional have been proposed for the KCIS correlation, we have tested in this work several combinations using already existing exchange functionals. It is worth to notice that these tests are essential because the arbitrary association between an exchange and a correlation functional, developed separately, can in principle lead to any kind of numerical results. In fact, the quality of exchange-correlation functionals is almost always due to quite unpredictable compensations of errors of each contribution. Among the most common GGA approaches, we have chosen the Perdew-Wang 91 (PW91) functional [29], the Perdew-Burke-Ernzerhof (PBE) form [15] and the widely-used Becke 88 (B) exchange [9]. Concerning the meta-GGA approach, only the exchange part of the Perdew-Kurth-ZupanBlaha (PKZB) model was tested [18].

Furthermore, the KCIS correlation functional has been also cast in a hybrid model of the general formula [30]:

$$
E_{x c}^{h y b}=a\left(E_{x}^{e x a c t}-E_{x}^{B}\right)+E_{x c}^{B K C I S}
$$

The constant $a$ can be fixed $a$ priori to $1 / 4$ by using theoretical considerations, or fitted to experimental data [30-32]. When using the Becke 88 exchange, the former approach using the 
theoretically determined value will be named B0KCIS, whereas, when using a fitted value for $a$, will be referred to as B1KCIS.

\section{Computational details}

All the DFT computations have been carried out within the unrestricted Kohn-Sham formalism as implemented in our development version of the Gaussian 99 package [33]. We have added the KCIS correlation functional, as well as its first and second derivatives with respect to all the required variables so that all the available exchange functionals as well as all the standard features of the package (as geometry optimization, computation of excitation energies, magnetic properties) can be used in conjunction with KCIS.

A number of different basis sets have been used [34]. In particular, the 6-311G $(d, p)$ basis set of Pople and co-workers has been used to optimize all the molecular structures, since previous experience showed that a polarized valence triple- $\zeta$ basis set generally provides nearly converged structural parameters by DFT methods. An extended basis set, namely the 6$311++\mathrm{G}(3 d f, 3 p d)$ one, has next been used to evaluate all the energetic parameters (atomization and dissociation energies). Finally, magnetic properties have been evaluated with the EPR-III basis set which has been purposely tailored for this kind of DFT applications.

When necessary, some computations have been also carried out with standard DFT model, such B3LYP, PBE or PBE0 [34].

\section{Results and discussion}

The validation of a functional is still a problematic ground, where some arbitrary (and limited) choices have to be done. As matter of fact, that it is not possible a priori to predict the reliability of a chosen functional form in chemical applications and an investigation is then compulsory before routine applications. For instance, the B3LYP functional can be considered as an enlightening example for the large number of papers assessing its validity (see for instance the test cases reported in reference 1). Even if a large part of functionals have been tested only on thermochemistry (using the standard G2 molecular data set), some of them have been the subjects of more detailed investigations on large number of already wellknown chemical systems or processes [7,10,11]. Following this latter viewpoint, we have tested the quality of results given by the new functionals including KCIS, concerning thermodynamics, geometrical, spectroscopic and magnetic properties on chemical systems including some traditional pathological cases for DFT methods. 


\subsection{Atomic correlation energies}

The first step for assessing the performances of the KCIS functional is to compared the correlation energies of the atoms belonging to the first two rows of the periodic table [35]. As can been seen by the results, obtained using HF densities, reported in table I, the accuracy of KCIS is at the same level than those of the most accurate correlation functionals, such as LYP, PKZB and PW91. This is a remarkable result, since these last functionals all contain parameters adjusted to experimental data, while this is not the case for the KCIS functional.

A part for this overall behaviour, two points must be stressed. First of all, it is noteworthy that the larger errors are obtained with atoms having an occupation of $p$ orbitals. Indeed, the correlation energy of this kind of electronic configurations often involved contributions from virtual states which should be localized in the gap of the excitation spectrum but which are totally neglected by KCIS [XX]. As second point, we notice that although all the functional give similar errors, the mean absolute errors (mae's) are different for the first and second rows, the KCIS and the LYP functionals providing a more balance description between first and second rows atoms.

\subsection{Atomization energies}

In order to carry out a detailed selection of the numerous models that can be build with KCIS, we have computed for each functional the mae's with respect to experimental values on atomization energies of the molecules belonging to the standard G2-1 (55 molecules) and the extended G2 (148 molecules) sets [36-38]. These sets can be considered as a DFT "classical" test, representatives of covalently bonded systems [39]. Compared to the G2-1 set which contains small saturated molecules, the G2 set includes bigger molecules, eventually nonsaturated.

The results collected in table II, show that, among the most common functionals, the reference is the hybrid B3LYP model, with a mae of $2.4 \mathrm{kcal} / \mathrm{mol}$ over the $\mathrm{G} 2-1$ set and 3.1 $\mathrm{kcal} / \mathrm{mol}$ for G2. In contrast, the PBE0 functional, which has no empirical parameters, leads to a relatively small mae for this kind of systems with $3.1 \mathrm{kcal} / \mathrm{mol}$ for the $\mathrm{G} 2-1$ set. The two most accurate model including the KCIS correlation are the PKZBKCIS and BKCIS with mae's of 4.1 and $8.2 \mathrm{kcal} / \mathrm{mol}$ over the G2-1 and G2 sets, respectively. The optimization of the parameter present in the exchange part of PKZB $(D=0.113)$ [18] leads to the PKZB $_{\text {opt KCIS functional }}\left(\mathrm{D}_{\mathrm{opt}}=0.104\right)$ which is not significantly better than the original PKZBKCIS (mae of $3.9 \mathrm{kcal} / \mathrm{mol}$ over the G2-1 set). Furthermore, the introduction of $25 \%$ of 
exact exchange (PKZB0KCIS) only deteriorates the quality of the results (mae of 6.9 $\mathrm{kcal} / \mathrm{mol}$ for G2-1). The construction of a more accurate model rather requires hybrid models with the BKCIS functional. In fact, upon introduction of $25 \%$ of HF exchange into this functional (leading to the BOKCIS model), the mae falls to $3.0 \mathrm{kcal} / \mathrm{mol}$ for $\mathrm{G} 2-1$. The optimization of the percentage of exact exchange over the G2-1 set (B1KCIS with $23.9 \%$ of exact exchange) does not induce significant improvements (see last line in table II). These good performances of B0KCIS, whereas the B0LYP functional is significantly less accurate, are, of course, to be credited to the different correlation.

As last test, we have carried out some computations on the G2 set, without the SCF procedure, using HF or PBE densities and only BOKCIS energy evaluation. This test is particularly interesting, since some recent implementations of exchange-correlation functionals do not include the derivatives need to the SCF procedure [6,18]. Our results (not reported in table II) show that with a PBE density the mae is $9.1 \mathrm{kcal} / \mathrm{mol}$ and it rises up to $11.7 \mathrm{kcal} / \mathrm{mol}$ with the $\mathrm{HF}$ densities. Both results are really far from the $3.0 \mathrm{kcal} / \mathrm{mol}$ obtained with the complete SCF procedure, thus warning about the use of auto-coherent procedure.

In the light of these first results, we have decided to study in details the performances of the KCIS functional, and especially those provided by the B0KICS model, which seems to be very accurate for covalent systems, even if it includes only one empirical parameter (in the exchange contribution). The natural reference to compare this model is, in our opinion, the similar B0LYP functional, but, in order to have a more complete picture, we consider also the popular B3LYP and PKZB functionals.

\subsection{Atomic total energies and geometry optimization}

As a first step, we have evaluated the total energies of some atoms, $\mathrm{H}$ through Ar. The results are collected in table III and are compared with accurate post-HF values (labeled as "exact" in the table) [33]. It is interesting to evaluate the accuracy of functionals concerning atomic total energies since this property has been recently added to a wide training set used to test and optimize DFT models [40]. Over these 18 atoms, B0KCIS provides a mae of 0.008 Hartree. This very good result are equaled only by BOLYP containing though four extra empirical parameters in the correlation contribution. We can also notice the astonishing accuracy of B0KCIS over the second row of the periodic table (mae of 0.004 Hartree).

After atomization and atomic energies, we have tested the ability of our new model to reproduce correct molecular geometrical parameters. Figure 1 reports the mae's over bond 
lengths of 32 molecules belonging to the G2 set computed with several functionals. With a mae of $0.008 \AA$, B0KCIS leads to about the same accuracy of other hybrid models PBE0, B3LYP and BOLYP. In fact, it is a well-known behavior: the introduction of a fraction of exact exchange in a functional generally leads to very accurate models concerning bond lengths. Indeed, since bond lengths are usually too short at the Hartree-Fock level and to long for GGA functionals, hybrid models benefit from a compensation of errors [31].

\subsection{Other tests}

Even if the exchange-correlation term to be approximated in the KS approach is usually decomposed in well-separated exchange and correlation parts, it is difficult to determine the dependence of one molecular properties on just one of these contributions. Few exceptions are represented by the van der Waals interactions and excitations dimers, both essentially ruled by the asymptotic behavior of the exchange functional [10,41]. Here, instead, we focused on more correlation-dependent properties/systems. In this context, small H-bonded dimers are an invaluable test, both for their chemical role and for the difficult to describe the subtle physical effects (electrostatic, charge transfer, polarization) present. It is therefore interesting to estimate the quality of DFT functionals on the kind of systems. Table VI collects geometrical parameters and interaction energies of three small H-bond dimers, namely $\left(\mathrm{H}_{2} \mathrm{O}\right)_{2},(\mathrm{HCl})_{2}$ and $(\mathrm{HF})_{2}$. The computed interaction energies have been corrected for BSSE error which has an order of magnitude of about $0.2 \mathrm{kcal} / \mathrm{mol}$ for the first two dimers with the large 6-311++G(3df,3pd) basis set, while this error is even lower for $(\mathrm{HF})_{2}$. For the three systems, only the global energy minimum, of $\mathrm{C}_{\mathrm{s}}$ symmetry, has been investigated, even if other stable rearrangements could actually exist [42].

The water dimer has been studied in considerable detail and the currently accepted values for the binding energy and the $\mathrm{OO}$ distance are $5.4 \pm 0.7 \mathrm{kcal} / \mathrm{mol}$ (not including ZPE corrections) and $2.952 \AA$, respectively $[43,44]$. Refined post-HF computations suggest that the interaction energy is close to $4.7 \mathrm{kcal} / \mathrm{mol}$ and give a shorter intermolecular distance $(2.925 \AA)$ [42,45]. As already well documented (see reference 1 for an almost complete bibliography), the equilibrium geometry of the water dimer is quite accurately reproduced by standard DFT methods, all the predicted OO distances falling between 2.87 and $2.91 \AA$, i.e. close to the MP2 estimate [1]. Our results are reported in table IV, and compared with experimental and post-HF data [42-44]. The most striking feature of these results is the B0KCIS distance, which is slightly longer $(2.91 \AA)$ than other DFT values, but closer to the experimental value $(2.95 \AA)$ [42]. At the same time the binding energy is slightly 
underestimated (3.8 vs. $4.7 \mathrm{kcal} / \mathrm{mol}$ for the best $a b$ initio value). Interesting both B3LYP and B0LYP provide higher interactions energies and shorter intermolecular distances, thus showing the correlation between these two quantities.

Similar trends are present for all the others two dimers, the B0KCIS providing always lower interactions energies and higher H-bridge distance than the B3LYP and B0LYP functionals. In the case of the hydrogen fluoride dimer, the combined effects of shorter distance/lower energy results in a better agreement with the experimental data (see table IV) [47]. This is not the case for $(\mathrm{HCl})_{2}$, where all the methods provide an poor accord with experiments [48]. A detailed comparison with literature data, suggests that more subtle effects, involving either exchange and correlation contributions, are at work in such H-bonded dimers [7].

The next test is represented by bimolecular nucleophilic substitution reactions $\left(S_{N} 2\right)$, which have an important place in organic chemistry. The symmetric Walden inversion, in particular, a excellent well-characterized example [49-54]:

$$
\mathrm{Cl}^{-}+\mathrm{CH}_{3} \mathrm{Cl} \longrightarrow \mathrm{ClCH}_{3}+\mathrm{Cl}^{-}
$$

This reaction has a double-well energetic profile, with two minima corresponding to the formation of a pre- and a post-reaction ion-molecule complex and a saddle point (SP) with $\mathrm{D}_{3 \mathrm{~h}}$ symmetry $\left(\left[\mathrm{Cl}{ }^{\cdots} \mathrm{CH}_{3}{ }^{\cdots} \mathrm{Cl}\right]^{-}\right)$. The most significant thermodynamic quantities are the complexation energy of the ion-molecule complex $\left(\Delta \mathrm{E}_{\text {comp }}\right)$, the activation energy, i.e. the relative energy of the $D_{3 h}$ saddle point with respect to the ion-molecule complex $\left(\Delta \mathrm{E}^{\#}\right)$, and the overall barrier $\left(\Delta \mathrm{E}_{\mathrm{ovr}}\right)$, defined as the difference between these two energies. The computed energies are collected in Table V. Apart from PBE0 which leads to a realistic reaction barrier, it is striking to notice that the more popular semi-empirical approaches, B3LYP or B0LYP, as well as more theoretically developed functionals, like PBE or PKZB, lead to zero or even negative values for $\Delta \mathrm{E}_{\mathrm{ovr}}$. Whereas the computation of initial closed-shell reagents does not generate particular difficulties, the determination of the energy of the charged transition state energy $\left[\mathrm{Cl}{ }^{\cdots} \mathrm{CH}_{3}{ }^{\cdots} \mathrm{Cl}\right]^{-}$by DFT approaches is more involved [52]. In fact, the majority of functionals are not corrected for the self-interaction error in the exchange part as well as in the correlation part, which implies an exaggerated delocalization of the electron density that over-stabilized the transition state [55]. On the contrary, the B0KCIS, which is corrected for self-interaction error in the correlation part, gives a too positive barrier (3.6 kcal/mol) but reproducing the trend of the MP2 computation $(2.5 \mathrm{kcal} / \mathrm{mol})$. The comparison between the results given by B0LYP and B0KCIS clearly shows the very strong influence of the correlation contribution for these applications. 
Proton transfer mechanisms are often of first importance in chemical or biological processes. In spite of the apparent simplicity of this phenomenon, the theoretical description of proton transfer is a difficult problem for both post-Hartree-Fock methods and DFT approaches. In particular, it is well-known that current functionals provide very low activation energies [5658]. The situation is even more involved when the motion of the proton is coupled with a rearrangement of the $\pi$ electronic system, as happens in malonaldehyde. In fact, the enol form of this molecule is stabilized by an intramolecular hydrogen bond, and it is possible to switch form a stable form to the other one by a proton transfer mechanism via a transition state of $\mathrm{C}_{2 \mathrm{v}}$ symmetry [57]. Table VI contains the most significant geometrical parameters as well as energetic barriers for the minimum and the saddle point of malonaldehyde. Whereas BOLYP over-estimates the energetic barrier with respect to the MP2 computations, (3.5 and 2.8 $\mathrm{kcal} / \mathrm{mol}$, respectively), B0KCIS gives geometrical and energetic parameters in good agreement with the reference values. Once again, the difference of $1 \mathrm{kcal} / \mathrm{mol}$ in the energetic barrier given by BOLYP and BOKCIS shows the importance of the correlation contribution and the better quality of KCIS over LYP for this kind of applications.

Finally, the last difficult playground is represented by one of the physical constant derived from EPR spectroscopic: the isotropic hyperfine coupling constant. The isotropic hyperfine coupling constant of a nucleus $n$, linked to the interaction of the nuclear spin with an electronic spin by the contact Fermi mechanism, depends on the electronic spin density $\rho^{s}\left(\mathbf{r}_{n}\right)$ at the position of the nucleus $\mathbf{r}_{n}$ [59]:

$$
a_{n}=\frac{8 \pi}{3 h} g_{e} \beta_{e} g_{n} \beta_{n} \rho^{s}\left(\mathbf{r}_{n}\right)
$$

where $\beta_{e}, \beta_{n}$ are the electronic and nuclear magnetons, $g_{e}, g_{n}$ the electronic and nuclear $\mathrm{g}$ factors, and $h$ is the Planck's constant. The spin density can be easily obtained from density matrices of electrons $\alpha$ and $\beta, P_{\mu, v}^{\alpha}$ et $P_{\mu, v}^{\beta}$, and from the basis atomic orbitals $\left\{\varphi_{\mu}\right\}$ :

$$
\rho^{s}\left(\mathbf{r}_{n}\right)=\sum_{\mu, \mathrm{v}}\left(P_{\mu, \mathrm{v}}^{\alpha}-P_{\mu, \mathrm{v}}^{\beta}\right) \varphi_{\mu}^{*}\left(\mathbf{r}_{n}\right) \varphi_{\mathrm{v}}\left(\mathbf{r}_{n}\right)
$$

This spin density on the nucleus can have two origins. The first direct origin is simply provided by a unpaired electron in an orbital which has a non-zero density at the position of the nucleus. The second indirect origin is due to spin polarization of paired electrons, which have a non-zero presence probability on the nucleus, caused by the interaction with the unpaired electron under the effect of electronic correlation which acts differently depending whether the electrons have the same spin or opposite ones [60]. 
In order to test B0KCIS, we have performed computations on the methyl and vinyl radicals. These radicals can be considered as prototype of $\pi$ and $\sigma$ organic radicals, respectively. The computed values of hyperfine coupling constants are collected in Table VII. First of all, it is striking to notice that for this property, DFT approaches are globally more accurate than very refined correlation $a b$ initio methods like CCSD (Coupled-Cluster Single Double) or CAS (Complete Active Space) [61]. For the methyl radical, the unpaired electron being in a $p$ orbital of zero-density on the carbon atom, the hyperfine coupling constants originate exclusively from spin polarization of electrons of the $2 s$ orbital for $\mathrm{C}$ or the $1 s$ orbital for $\mathrm{H}$. The same phenomenon appears in the allyl radical. Concerning electrons of same spin, this interaction can only be treated properly with an accurate exchange functional like in PBE0 [62]. On the contrary, when a less accurate exchange functional is used like $\mathrm{B}$, results strongly depend on error compensation with the correlation part. As a consequence, B0LYP overestimates the hyperfine coupling constant of $\mathrm{C}$ in the methyl radical whereas B0KCIS underestimates it.

The case of non-saturated $\sigma$ radicals like the vinyl radical is more complicated to treat by DFT approaches as well as by post-HF methods since it involves both direct and indirect contributions to hyperfine coupling constants [60]. Nevertheless, it is striking to notice the excellent value of hyperfine coupling constant of $\mathrm{C}_{\alpha}$, dominated by the direct contribution of the unpaired electron in a $\sigma$ orbital of non-zero density on the nucleus, given by B0KCIS. The comparison of the results given by BOLYP and B0KCIS functionals suggests the importance of the correlation contribution for this application.

\section{Conclusions}

In this work, we have tested in a systematic way the new meta-GGA parameter-free correlation functional KCIS. Concerning atomic correlation energies, this functional is globally more accurate than other theoretical or semi-empirical correlation functionals like the correlation part of PBE or LYP, respectively. The search for the best exchange functional which can been coupled to KCIS has leaded to a new hybrid model with the Becke 88 (B) exchange functional: B0KCIS. This model, including $25 \%$ of exact exchange and containing only one empirical parameter in the exchange part, has been tested over a varied set of physico-chemical properties and have turned out to have performances better or at least equivalent to those provided by semi-empirical exchange-correlation functionals like B3LYP. 
In details, the use of BOKCIS instead of BOLYP brings a significant improvement for properties where the correlation contribution plays an important role like for atomization energies, energetic reaction barriers and magnetic properties.

While, in the present study we have searched for the best suitable exchange functional, among those already available "on the market", to be used with KCIS, we believe that it would be interesting to developed a new exchange functional entirely adapted to KCIS in order to obtain an even more accurate model. Work is in progress in such a direction. 


\section{References}

[1] Kock, W.;Holthausen, M.C. A Chemist's Guide to Density Functional Theory, (Weinheim Wiley-VCH 2000).

[2] R.G. Parr and W. Yang, Density Functional Theory of Atoms and Molecules (Oxford University Press, New York, 1989).

[3] K. Burke, J.P. Perdew and Y. Wang, in Electronic density functional theory: recent progress and new derivations, edited by J.F. Dobson, G. Vignale and M.P. Das (Plenum Press, New York, 1997).

[4] C. Adamo, A. di Matteo, and V. Barone, Adv. Quantum Chem. 36 (1999) 4.

[5] S. Kurth, J.P. Perdew and P. Blaha, Int. J. Quantum Chem. 75 (1999) 889

[6] J. Tao, J. Chem. Phys. 116 (2002) 2335.

[7] C. Adamo and V. Barone, J. Chem. Phys 116 (2002) 5933.

[8] A.D. Becke, J. Chem. Phys. 85, 7184 (1986).

[9] A.D. Becke, Phys. Rev. A 38, 3098 (1988)

[10] C. Adamo and V. Barone, J. Chem. Phys. 108, 664 (1998).

[11] W.M. Hoe, A.J. Cohen and N.C. Handy, Chem. Phys. Lett. 341, 319 (2001)

[12] T. Tsuneda and K. Hirao, Phys. Rev. B 62, 15527 (2000)

[13] J.P. Perdew and Y. Wang, Phys. Rev. B 33, 8800 (1986).

[14] PW91 correlation

[15] J.P. Perdew, K. Burke and M. Ernzerhof, Phys. Rev. Lett. 77 (1996) 3865, ibid 78 (1997) $1396(\mathrm{E})$.

[16] T. van Voorhis and G.E. Scuseria, J. Chem. Phys. 109 (1998) 400.

[17] Becke mGGA

[18] J.P. Perdew, S. Kurth, A. Zupan and P. Blaha, Phys. Rev. Lett. 82 (1999) 2544.

[19] E. Proynov, H. Chermette and D.R. salahub, J. Chem. Phys. 113 (2000) 10013.

[20] R. Colle and O. Salvetti, Theor. Chim. Acta 37 (1975) 329.

[21] C. Lee, W. Yang and R.G. Parr, Phys. Rev. B 37 (1988) 785.

[22] T. Tsuneda, T. Suzumura and K. Hirao, J. Chem. Phys. 110 (1999) 10664

[23] Y. Imamura, G.E. Scuseria and R.M. Martin, J. Chem. Phys. 116 (2002) 6458.

[24] J.B. Krieger, J. Chen, G.J. Iafrate and A. Savin in Electron Correlations and Materials Properties, A. Gonis and N. Kioussis (Eds) Plenum, New York (1999).

[25] J. Rey and A. Savin, Int. J. Quantum Chem. 69 (1998) 581. 
[26] J.B. Krieger, J. Chen and S. Kurth, in Density functional theory and its applications to materials, ed. by V. van Doren, C. van Alsenoy, P. Geerlings, AIP Conference Proceedings, Vol. 577, 48 (2001)

[27] A.D. Becke, J. Chem. Phys. 98 (1993) 5648.

[28] A.D. Becke, J. Chem. Phys. 109, 2092 (1998).

[29] PW exchange

[30] Becke one parameter hybrid

[31] C. Adamo and V. Barone, Chem. Phys. Lett. 272, 242 (1997).

[32] A.D. Becke, J. Chem. Phys. 109, 5648 (1998).

[33] M. J. Frisch, G. W. Trucks, H. B. Schlegel, G. E. Scuseria, M. A. Robb, J. R. Cheeseman, V. G. Zakrzewski, J. A. Montgomery, Jr., R. E. Stratmann, J. C. Burant, S. Dapprich, J. M. Millam, A. D. Daniels, K. N. Kudin, M. C. Strain, O. Farkas, J. Tomasi, V. Barone, M. Cossi, R. Cammi, B. Mennucci, C. Pomelli, C. Adamo, S. Clifford, J. Ochterski, G. A. Petersson, P. Y. Ayala, Q. Cui, K. Morokuma, D. K. Malick, A. D. Rabuck, K. Raghavachari, J. B. Foresman, J. Cioslowski, J. V. Ortiz, B. B. Stefanov, G. Liu, A. Liashenko, P. Piskorz, I. Komaromi, R. Gomperts, R. L. Martin, D. J. Fox, T. Keith, M. A. Al-Laham, C. Y. Peng, A. Nanayakkara, C. Gonzalez, M. Challacombe, P. M. W. Gill, B. Johnson, W. Chen, M. W. Wong, J. L. Andres, C. Gonzalez, M. Head-Gordon, E. S. Replogle, and J. A. Pople, Gaussian 99 Development version (Revision B.05), Gaussian Inc.: Pittsburgh PA, 1999

[34] Æ. Frisch and M.J. Frisch, Gaussian 98 User's Reference (Gaussian, Inc., Pittsburgh 1998) and reference therein

[35] S.J. Chakravorty, S.R. Gwaltney, E.R. Davidson, F.A. Parpia and C.F. Froese Fischer, Phys. Rev. A 47, 3649 (1993)

[36] J. A. Pople, M. Head-Gordon, D.J. Fox, K. Raghavachari and L.A. Curtiss, J. Chem. Phys. 90, 5622 (1989).

[37] L.A. Curtiss, C. Jones, G.W. Trucks, K. Raghavachari and J. A. Pople J. Chem. Phys. 93, 2537 (1990).

[38] L.A. Curtiss, K. Raghavachari, P.C. Redfern and J. A. Pople J. Chem. Phys. 106, 1063 (1997).

[39] B. G. Johnson, P.M.W. Gill and J. A. Pople, J. Chem. Phys. 98, 5612 (1993).

[40] G. Kin-Lic Chan and N.C. Handy; J. Chem. Phys. 112, 5639 (2000)

[41] J.M. Pérez-Jordà, A.D. Becke, Chem. Phys. Lett. 233, 134 (1995).

[42] A.K. Rappé and E.R. Bernstein, J. Phys. Chem. A, 104, 6117 (2000). 
[43] L.A. Curtis, D.J. Frurip and M. Blander, J. Chem. Phys. 71, 2073 (1979).

[44] J.A. Odutola and T.R. Dyke, J. Chem. Phys. 72, 5062 (1980).

[45] M.J. Frisch, J.E. Del Bene, J.S. Binkley and H.F. Schaefer III, J. Chem. Phys. 84, 2279 (1986).

[46] C. Tuma, A.D. Boese and N.C. Handy, Phys. Chem. Chem. Phys. 1, 3939 (1999).

[47] A.S. Pine and B. Howard, J. Chem. Phys. 84, 590 (1986).

[48] M. Quack and M.A. Suhm, Theor. Chem. Acta 93, 61 (1996) and references therein

[49] S.S. Shaik, H.B. Schlegel and S. Wolfe, Theoretical aspects of physical organic chemistry. The $\mathrm{SN}_{2}$ mechanism.(Wiley, New York, 1992).

[50] T.N. Truong and E.V. Stefanovich, J. Phys. Chem. 99, 14700 (1995).

[51] M.N. Glukhovtsev, A. Pross and L. Radom, J. Am. Chem. Soc. 117, 2024 (1995).

[52] M.N. Glukhovtsev, R.D. Bach, A. Pross and L. Radom, Chem. Phys. Lett. 260, 558 (1996).

[53] J.W. Larson and T.B. McMahon, J. Am. Chem. Soc. 106, 517 (1984).

[54] S.E. Barlow, J.M. van Doren and V.M. Bierbaum, J. Am. Chem. Soc.116, 10645 (1994)

[55] Ziegler - SIC

[56] V. Barone, L. Orlandini and C. Adamo, Chem. Phys. Lett. 231, 295 (1994).

[57] V. Barone and C. Adamo, J. Chem. Phys. 105, 11007 (1996).

[58] S. Sadhukhan, D. Munoz, C. Adamo and G.E. Scuseria, , Chem. Phys. Lett. 306, 83 (1999).

[59] W. Weltner, Jr., Magnetic Atoms and Molecules, Dover, New York (1989).

[60] C. Adamo, V. Barone, A. Fortunelli, J. Chem. Phys. 102, 384 (1995).

[61] V. Barone, V In Recent Advances in Density Functional Methods Part 1, Chong, D.P. Ed.; World Scientific Publishing Co.; Singapore (1995, p. 287).

[62 ] C. Adamo, M. Cossi and V. Barone, J. Mol. Struct. (THEOCHEM) 493, 145 (1999). 
Table I. Exact correlation energies of atoms belonging to the first two rows of the periodic table and differential energies (Hartree) computed with the 6-311+G(3df,3pd) basis set and HF densities.

\begin{tabular}{ccccccc}
\hline Atoms & Exact $^{\mathrm{a}}$ & PBE & PW91 & LYP & PKZB & KCIS \\
\hline $\mathrm{H}$ & 0.000 & -0.006 & -0.007 & 0.000 & 0.000 & 0.000 \\
$\mathrm{He}$ & -0.042 & 0.000 & -0.004 & -0.002 & -0.005 & 0.001 \\
$\mathrm{Li}$ & -0.045 & -0.006 & -0.012 & -0.008 & -0.009 & -0.004 \\
$\mathrm{Be}$ & -0.094 & 0.009 & 0.000 & 0.000 & 0.001 & 0.008 \\
$\mathrm{~B}$ & -0.125 & 0.013 & 0.001 & 0.000 & 0.008 & 0.014 \\
$\mathrm{C}$ & -0.156 & 0.012 & -0.002 & -0.002 & 0.008 & 0.013 \\
$\mathrm{~N}$ & -0.188 & 0.009 & -0.009 & -0.004 & 0.005 & 0.008 \\
$\mathrm{O}$ & -0.258 & 0.023 & 0.001 & 0.001 & 0.017 & 0.020 \\
$\mathrm{~F}$ & -0.325 & 0.033 & 0.006 & 0.003 & 0.022 & 0.023 \\
$\mathrm{Ne}$ & -0.391 & 0.040 & 0.009 & 0.007 & 0.028 & 0.024 \\
$\mathrm{MAE}(\mathrm{H}-\mathrm{Ne})$ & -- & 0.015 & 0.005 & 0.003 & 0.010 & 0.012 \\
& & & & & & \\
$\mathrm{Na}$ & -0.396 & 0.024 & -0.011 & -0.013 & 0.013 & 0.005 \\
$\mathrm{Mg}$ & -0.438 & 0.027 & -0.012 & -0.021 & 0.013 & 0.002 \\
$\mathrm{Al}$ & -0.470 & 0.024 & -0.019 & -0.024 & 0.012 & -0.001 \\
$\mathrm{Si}$ & -0.505 & 0.021 & -0.026 & -0.024 & 0.009 & -0.006 \\
$\mathrm{P}$ & -0.540 & 0.014 & -0.037 & -0.026 & 0.003 & -0.014 \\
$\mathrm{~S}$ & -0.605 & 0.021 & -0.035 & -0.023 & 0.008 & -0.009 \\
$\mathrm{Cl}$ & -0.666 & 0.022 & -0.038 & -0.024 & 0.007 & -0.013 \\
$\mathrm{Ar}$ & -0.722 & 0.016 & -0.048 & -0.028 & -0.001 & -0.023 \\
MAE (Na-Ar) & -- & 0.021 & 0.028 & 0.023 & 0.008 & 0.009 \\
Total MAE & -- & 0.018 & 0.015 & 0.012 & 0.009 & 0.011
\end{tabular}

a) ref. 35 
Table II. Mean absolute errors (MAE) and maximal errors $(\mathrm{kcal} / \mathrm{mol})$ for the atomization energies of molecules belonging to the G2-1 (55 molecules) and G2 (148 molecules), computed with the $6-311+\mathrm{G}(3 \mathrm{df}, 2 \mathrm{p})$ basis set and MP2/6-31G* geometries.

\begin{tabular}{ccccc}
\hline & \multicolumn{2}{c}{$\mathrm{G} 2-1$} & G2 \\
& MAE & $\max$. & MAE & max. \\
\hline PBE & 8.2 & $29.1\left(\mathrm{CO}_{2}\right)$ & 17.2 & $50.5\left(\mathrm{C}_{2} \mathrm{~F}_{2}\right)$ \\
PKZB & 3.6 & $11.0\left(\mathrm{O}_{2}\right)$ & 4.5 & $-37.7\left(\mathrm{SiF}_{4}\right)$ \\
PBE0 & 3.1 & $-10.7\left(\mathrm{SiO}^{2}\right)$ & 5.0 & $-21.7\left(\mathrm{SiF}_{4}\right)$ \\
B3LYP & 2.4 & $-9.9\left(\mathrm{SO}_{2}\right)$ & 3.1 & $-20.2\left(\mathrm{SiF}_{4}\right)$ \\
B0LYP & 5.1 & $-20.6\left(\mathrm{SO}_{2}\right)$ & 11.2 & $-30.1\left(\mathrm{SiF}_{4}\right)$ \\
& & & & \\
PBEKCIS & 11.9 & $35.2\left(\mathrm{CO}_{2}\right)$ & 24.9 & $66.5\left(\mathrm{C}_{5} \mathrm{H}_{5} \mathrm{~N}\right)$ \\
PKZBKCIS & 4.1 & $-14.4\left(\mathrm{C}_{2} \mathrm{H}_{6}\right)$ & 9.7 & $38.8\left(\mathrm{SiF}_{4}\right)$ \\
PKZB & $13.7\left(\mathrm{O}_{2}\right)$ & 10.0 & $-23.2\left(\mathrm{C}_{4} \mathrm{H}_{10}\right)$ \\
BKCIS & 3.9 & $25.9\left(\mathrm{CO}_{2}\right)$ & 14.6 & $39.6\left(\mathrm{NO}_{2}\right)$ \\
B0KCIS & 8.2 & $-10.5\left(\mathrm{SO}_{2}\right)$ & 5.3 & $-28.6\left(\mathrm{SiF}_{4}\right)$ \\
B1KCIS & 3.0 & $-9.6\left(\mathrm{SiO}_{1}\right)$ & 5.4 & $-28.1\left(\mathrm{SiF}_{4}\right)$ \\
\hline \hline
\end{tabular}


Table III. Exact total atomic energies and differential SCF energies (Hartree), computed with the $6-311+\mathrm{G}(3 d f, 3 p d)$ basis set.

\begin{tabular}{cccccccc}
\hline Atoms & Exact $^{\text {a }}$ & PBE & PBE0 & B3LYP & B0LYP & PKZB & B0KCIS \\
\hline H & -0.500 & 0.000 & -0.001 & -0.002 & 0.002 & 0.004 & 0.002 \\
$\mathrm{He}$ & -2.904 & 0.014 & 0.011 & -0.009 & 0.000 & 0.002 & 0.003 \\
$\mathrm{Li}$ & -7.478 & 0.018 & 0.012 & -0.012 & -0.003 & 0.004 & 0.002 \\
$\mathrm{Be}$ & -14.667 & 0.041 & 0.032 & -0.004 & 0.006 & 0.019 & 0.015 \\
$\mathrm{~B}$ & -24.654 & 0.045 & 0.037 & -0.009 & 0.003 & 0.036 & 0.017 \\
$\mathrm{C}$ & -37.845 & 0.052 & 0.042 & -0.012 & 0.000 & 0.056 & 0.015 \\
$\mathrm{~N}$ & -54.589 & 0.060 & 0.049 & -0.012 & 0.002 & 0.083 & 0.015 \\
$\mathrm{O}$ & -75.067 & 0.062 & 0.054 & -0.024 & -0.008 & 0.110 & 0.011 \\
$\mathrm{~F}$ & -99.734 & 0.073 & 0.067 & -0.028 & -0.010 & 0.147 & 0.011 \\
$\mathrm{Ne}$ & -128.938 & 0.092 & 0.087 & -0.022 & -0.001 & 0.198 & 0.018 \\
$\mathrm{MAE}(\mathrm{H}-\mathrm{Ne})$ & -- & 0.046 & 0.039 & 0.013 & 0.004 & 0.066 & 0.011 \\
$\mathrm{Na}$ & -162.255 & 0.099 & 0.085 & -0.032 & -0.013 & 0.230 & 0.006 \\
$\mathrm{Mg}$ & -200.053 & 0.108 & 0.092 & -0.040 & -0.023 & 0.267 & 0.001 \\
$\mathrm{Al}$ & -242.346 & 0.119 & 0.098 & -0.041 & -0.024 & 0.308 & -0.001 \\
$\mathrm{Si}$ & -289.359 & 0.134 & 0.108 & -0.035 & -0.020 & 0.351 & -0.001 \\
$\mathrm{P}$ & -341.259 & 0.155 & 0.124 & -0.023 & -0.008 & 0.401 & 0.005 \\
$\mathrm{~S}$ & -398.110 & 0.170 & 0.135 & -0.024 & -0.010 & 0.446 & 0.005 \\
$\mathrm{Cl}$ & -460.148 & 0.189 & 0.150 & -0.020 & -0.006 & 0.495 & 0.007 \\
$\mathrm{Ar}$ & -527.540 & 0.209 & 0.165 & -0.014 & 0.001 & 0.545 & 0.007 \\
MAE (Na-Ar) & -- & 0.148 & 0.120 & 0.029 & 0.013 & 0.380 & 0.004 \\
Total MAE & & & & & & & \\
& -- & 0.091 & 0.075 & 0.020 & 0.008 & 0.206 & 0.008
\end{tabular}

a) ref. 35 
Table IV. Dissociation energies $(\mathrm{kcal} / \mathrm{mol})$ and main geometrical parameters $(\AA)$ for H-bond dimers. The energies (corrected for BSSE error) have been computed with the 6$311++\mathrm{G}(3 \mathrm{df}, 3 \mathrm{pd})$ basis set with the $6-311 \mathrm{G}(\mathrm{d}, \mathrm{p})$ geometries

\begin{tabular}{|c|c|c|c|c|c|c|}
\hline & B3LYP & BOLYP & PKZB & B0KCIS & $\begin{array}{c}\text { Best } \\
\text { ab initio }\end{array}$ & Exp. $^{b}$ \\
\hline $\begin{array}{c}\left(\mathbf{H}_{\mathbf{2}} \mathbf{O}\right)_{2} \\
\mathrm{~d}(\mathrm{OO})\end{array}$ & 2.888 & 2.892 & 2.883 & 2.912 & 2.925 & 2.952 \\
\hline $\mathrm{d}\left(\mathrm{H}^{*} \mathrm{O}\right)$ & 1.926 & 1.931 & 2.364 & 1.952 & & \\
\hline$\Delta \mathrm{E}$ & 4.6 & 4.5 & 1.9 & 3.8 & 4.7 & $5.4 \pm 0.7$ \\
\hline $\begin{array}{l}(\mathbf{H F})_{2} \\
\mathrm{~d}(\mathrm{FF})\end{array}$ & 2.707 & 2.710 & 2.888 & 2.734 & 2.76 & $2.73 / 2.74$ \\
\hline $\begin{array}{c}\mathrm{d}(\mathrm{H} \cdot \mathrm{F}) \\
\Delta \mathrm{E}\end{array}$ & $\begin{array}{c}1.780 \\
4.7\end{array}$ & $\begin{array}{c}1.786 \\
4.7\end{array}$ & $\begin{array}{c}1.956 \\
3.0\end{array}$ & $\begin{array}{c}1.818 \\
3.8\end{array}$ & 4.6 & 4.2 \\
\hline $\begin{array}{r}(\mathbf{H C l})_{2} \\
\mathrm{~d}(\mathrm{ClCl}) \\
\mathrm{d}\left(\mathrm{H}^{*} \mathrm{Cl}\right)\end{array}$ & $\begin{array}{l}3.917 \\
2.626\end{array}$ & $\begin{array}{l}3.947 \\
2.657\end{array}$ & $\begin{array}{l}4.387 \\
3.093\end{array}$ & $\begin{array}{l}4.009 \\
2.724\end{array}$ & 3.790 & $3.75 / 3.79$ \\
\hline$\Delta \mathrm{E}$ & 1.2 & 0.9 & 0.8 & 0.8 & 2.0 & $1.4 / 2.0$ \\
\hline
\end{tabular}

a) ref. $43,45,46$; b) ref. $44,47,48$. 
Table V. Complexation energies of the ion-molecule complex $\left(\Delta \mathrm{E}_{\mathrm{comp}}\right)$, activation energies $\left(\Delta \mathrm{E}^{\#}\right)$ and energetic barriers relative to reagents $\left(\Delta \mathrm{E}_{\mathrm{r}}\right)$ for the considered $\mathrm{S}_{\mathrm{N}} 2$ reaction, computed at different levels of approximation. All the energies ( $\mathrm{kcal} / \mathrm{mol})$ have been computed with the $6-311++\mathrm{G}(3 \mathrm{df}, 3 \mathrm{pd})$ base set and the $6-311+\mathrm{G}(\mathrm{d}, \mathrm{p})$ geometries

\begin{tabular}{ccccccc}
\hline \hline & G2+(MP2) & B3LYP & B0LYP & PKZB & B0KCIS & Exp. \\
\hline$\Delta \mathrm{E}_{\text {comp }}$ & 10.6 & 9.8 & 9.7 & 9.6 & 8.4 & $12.2 \pm 2$ \\
$\Delta \mathrm{E}^{\#}$ & 13.0 & 9.1 & 9.7 & 9.6 & 11.9 & $13.3 \pm 2$ \\
$\Delta \mathrm{E}_{\mathrm{r}}$ & 2.5 & -0.7 & 0.0 & 0.0 & 3.6 & $1 \pm 1$
\end{tabular}


Table VI. Main geometrical parameters $(\AA)$ of the H-bond and relative energies $(\mathrm{kcal} / \mathrm{mol})$ for the minimum (M) and the saddle point (SP) of malonaldehyde. The energies have been computed with the $6-311++\mathrm{G}(3 d f .3 p d)$ basis set with the 6-311G $(d . p)$ geometries.

\begin{tabular}{ccccccccc}
\hline & \multicolumn{2}{c}{ MP2 } & \multicolumn{2}{c}{ B3LYP } & \multicolumn{2}{c}{ B0LYP } & \multicolumn{2}{c}{ B0KCIS } \\
& M & SP & M & SP & M & SP & M & SP \\
\hline & & & & & & & & \\
$\mathrm{d}(\mathrm{OO})$ & 2.581 & 2.355 & 2.580 & 2.367 & 2.593 & 2.363 & 2.564 & 2.356 \\
$\mathrm{~d}(\mathrm{H} \cdot \mathrm{O})$ & 1.678 & 1.197 & 1.687 & 1.208 & 1.711 & 1.207 & 1.666 & 1.202 \\
$\mathrm{~d}(\mathrm{OH})$ & 0.991 & 1.197 & 0.999 & 1.208 & 0.992 & 1.207 & 0.996 & 1.202 \\
$\Delta \mathrm{E}$ & 0.0 & 2.8 & 0.0 & 3.0 & 0.0 & 3.5 & 0.0 & 2.5 \\
\hline \hline
\end{tabular}


Table VII. Isotropic hyperfine coupling constants (a. Gauss) for the methyl and vinyl radical computed with the EPR-III basis set.

\begin{tabular}{|c|c|c|c|c|c|c|}
\hline & & B3LYP & B0LYI & 30KCIS & CCSD(T) & Exp. \\
\hline \multirow[t]{2}{*}{ methyl } & $a(C)$ & 29.8 & 31.7 & 24.9 & 27.8 & 28.4 \\
\hline & $\mathrm{a}(\mathrm{H})$ & -23.3 & -24.3 & -26.2 & -24.6 & -25.1 \\
\hline \multirow[t]{5}{*}{ vinyl } & $\mathrm{a}\left(\mathrm{C}_{\mathrm{a}}\right)$ & 113.1 & 112.7 & 107.6 & 121 & 107.6 \\
\hline & $\mathrm{a}\left(\mathrm{C}_{\mathrm{b}}\right)$ & -5.8 & -5.8 & -5.2 & -8 & -8.6 \\
\hline & $\mathrm{a}\left(\mathrm{H}_{\mathrm{a}}\right)$ & 17.8 & 17.7 & 12.3 & 9 & 13.3 \\
\hline & $\mathrm{a}\left(\mathrm{H}_{\mathrm{anti}}\right)$ & 41.9 & 41.9 & 40.7 & 28 & 34.2 \\
\hline & $\mathrm{a}\left(\mathrm{H}_{\text {syn }}\right)$ & 65.6 & 65.5 & 62.2 & 48 & 68.5 \\
\hline MAE & & 3.8 & 3.9 & 3.3 & 6.6 & -- \\
\hline
\end{tabular}




\section{Figure Captions}

Figure 1. Mean absolute errots (MAEs) for the bond lengths of 32 molecules belonging to the G2 set. computed with the 6-311G(d.p) basis set

Figure 2 Schematic structures and atom labelling of the methyl and vinyl radicals.

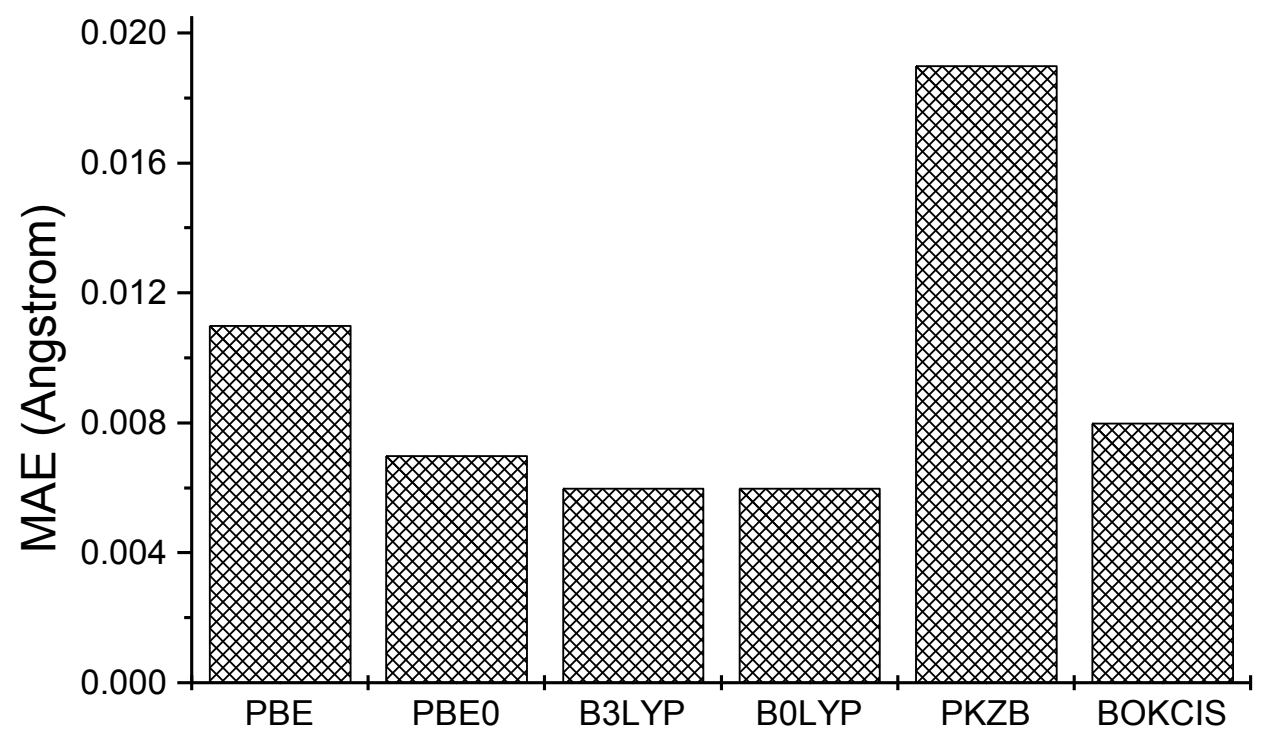

Figure 1 


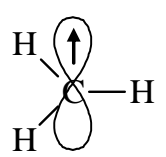

methyl

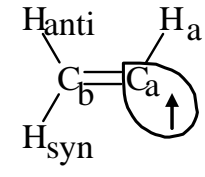

vinyl

Figure 2 


\section{Appendix: Expression of the KCIS correlation functional}

This appendix contains the complete expression of the KCIS correlation functional. The following seven variables are involved in this meta-GGA functional: $\rho_{\alpha}, \rho_{\beta}, \gamma_{\alpha}=g_{\alpha}{ }^{2}=\left|\nabla \rho_{\alpha}\right|^{2}, \gamma_{\beta}=g_{\beta}{ }^{2}=\left|\nabla \rho_{\beta}\right|^{2}$, $\gamma_{\alpha \beta}=\nabla \rho_{\alpha} \cdot \nabla \rho_{\beta}, \tau_{\alpha}$ and $\tau_{\beta}$ :

$$
E_{c}^{K C I S}\left[\rho_{\alpha}, \rho_{\beta}\right]=\int F\left(\rho_{\alpha}, \rho_{\beta}, \gamma_{\alpha}, \gamma_{\beta}, \gamma_{\alpha \beta}, \tau_{\alpha}, \tau_{\beta}\right) d \mathbf{r}
$$

where $F$ presents the following Self-Interaction Corrected form:

$$
F=\rho \varepsilon_{c}^{G G A G A P}\left(\rho_{\alpha}, \rho_{\beta}, g, \zeta\right)-\sum_{\sigma=\alpha, \beta} \frac{\tau_{\sigma}^{W}\left(\rho_{\sigma}, g_{\sigma}\right)}{\tau_{\sigma}} \rho_{\sigma} \varepsilon_{c}^{G G A G A P}\left(\rho_{\sigma}, 0, g_{\sigma}, \zeta=1\right)
$$

with $\rho=\rho_{\alpha}+\rho_{\beta}, g=|\nabla \rho|=\left(\gamma_{\alpha}+\gamma_{\beta}+2 \gamma_{\alpha \beta}\right)^{1 / 2}, \zeta=\frac{\rho_{\alpha}-\rho_{\beta}}{\rho}$ and $\tau_{\sigma}^{W}=\frac{g_{\sigma}^{2}}{8 \rho_{\sigma}}$.

$\varepsilon_{c}^{G G A G A P}$ is the correlation energy per particle of a uniform electron gas with a gap and including some gradient corrections. It can be obtained for any spin polarization $\zeta$ by the formula:

$$
\varepsilon_{c}^{G G A G A P}\left(\rho_{\alpha}, \rho_{\beta}, g, \zeta\right)=\varepsilon_{c}^{G A P, u n p}(\rho, g)+f(\zeta)\left[\varepsilon_{c}^{G A P, p o l}(\rho, g)-\varepsilon_{c}^{G A P, \text { unp }}(\rho, g)\right]
$$

with the polarization function:

$$
f(\zeta)=\frac{(1+\zeta)^{4 / 3}+(1-\zeta)^{4 / 3}-2}{2\left(2^{1 / 3}-1\right)}
$$

and the correlation energies of the unpolarized and polarized electron gas:

$$
\left\{\begin{array}{l}
\varepsilon_{c}^{G A P, \text { unp }}(\rho, g)=\frac{\varepsilon_{c}^{G G A, \text { unp }}\left(r_{s}, g\right)+c_{1, \text { unp }}\left(r_{s}\right) G(\rho, g)}{1+c_{2, \text { unp }}\left(r_{s}\right) G(\rho, g)+c_{3, \text { unp }}\left(r_{s}\right) G(\rho, g)^{2}} \\
\varepsilon_{c}^{G A P, \text { pol }}(\rho, g)=\frac{\varepsilon_{c}^{G G A, \text { pol }}\left(r_{s}, g\right)+c_{1, \text { pol }}\left(r_{s}\right) G(\rho, g)}{1+c_{2, \text { pol }}\left(r_{s}\right) G(\rho, g)+c_{3, \text { pol }}\left(r_{s}\right) G(\rho, g)^{2}}
\end{array}\right.
$$

where the Seitz local radius $r_{s}=\left(\frac{3}{4 \pi \rho}\right)^{1 / 3}$ and the gap $G(\rho, g)=\frac{g^{2}}{8 \rho^{2}}$ have been introduced.

The coefficients $c_{1, \text { unp }}\left(r_{s}\right), c_{2, \text { unp }}\left(r_{s}\right)$ and $c_{3, \text { unp }}\left(r_{s}\right)$ have been determined by a fitting procedure, starting from a model of uniform electron gas with a gap $G$ :

$$
c_{1, \text { unp }}=C \frac{2\left(\varepsilon_{c}^{\prime}\right)^{2}-\varepsilon_{c}^{0} \varepsilon_{c}^{\prime \prime}}{2\left(C \varepsilon_{c}^{\prime}-\varepsilon_{c}^{0^{2}}\right)}, c_{2, \text { unp }}=\frac{2 \varepsilon_{c}^{0} \varepsilon_{c}^{\prime}-C \varepsilon_{c}^{\prime \prime}}{2\left(C \varepsilon_{c}^{\prime}-\varepsilon_{c}^{0^{2}}\right)} \text { and } c_{3, \text { unp }}=-\frac{2\left(\varepsilon_{c}^{\prime}\right)^{2}-\varepsilon_{c}^{0} \varepsilon_{c}^{\prime \prime}}{2\left(C \varepsilon_{c}^{\prime}-\varepsilon_{c}^{0^{2}}\right)}
$$

with $C=0,06483 k_{F}{ }^{2}$ obtained from second-order perturbation theory, and the Fermi wave-vector $k_{F}=\left(\frac{9 \pi}{4}\right)^{1 / 3} \frac{1}{r_{s}}=\left(3 \pi^{2} \rho\right)^{1 / 3}$.

$\varepsilon_{c}^{0}$ is the correlation energy per particle of the unpolarized uniform electron gas at $G=0$, computed using the local part of the Perdew-Wang 91 (PW91) correlation functional:

$$
\varepsilon_{c}^{0}=\varepsilon_{c}^{P W 91 L c}\left(r_{s}, \zeta=0\right)
$$


$\varepsilon_{c}^{\prime}$ and $\varepsilon_{c}^{\prime \prime}$, are respectively the first and second derivatives of $\varepsilon_{c}$ with respect to $G$ at $G=0$, and can be calculated by the expressions:

$$
\varepsilon_{c}^{\prime} \approx \frac{a_{1} r_{s}^{3 / 2}}{1+a_{2} r_{s}^{1 / 2}+a_{3} r_{s}+a_{1} r_{s}^{3 / 2}}
$$

and

$$
\varepsilon_{c}^{\prime \prime} \approx \sum_{i=3}^{7} b_{i} r_{s}^{i}
$$

with $\quad a_{1}=0,04953 ; \quad a_{2}=1,07924 ; \quad a_{3}=0,07928 ; \quad b_{3}=-0,02504 ; \quad b_{4}=0,007026$; $b_{5}=-0,001268 ; b_{6}=0,0001136 ; b_{7}=-0,000003842$.

The coefficients $c_{1, p o l}\left(r_{s}\right), c_{2, p o l}\left(r_{s}\right)$ and $c_{3, p o l}\left(r_{s}\right)$ which are evolved in the polarized case are simply evaluated from the coefficients of the unpolarized case:

$$
c_{1, \text { pol }}\left(r_{s}\right)=0,7 c_{1, \text { unp }}\left(r_{s}\right), c_{2, \text { pol }}\left(r_{s}\right)=1,5 c_{2, \text { unp }}\left(r_{s}\right) \text { and } c_{3, \text { pol }}\left(r_{s}\right)=2,59 c_{3, \text { unp }}\left(r_{s}\right)
$$

The gradient correction in the correlation energies per particle of the unpolarized and polarized electron gas, $\varepsilon_{c}^{G G A, u n p}$ and $\varepsilon_{c}^{G G A, p o l}$, is introduced by the expressions:

$$
\left\{\begin{array}{l}
\varepsilon_{c}^{G G, \text { unp }}\left(r_{s}, g\right)=\frac{\varepsilon_{c}^{0}}{1+\beta \ln \left(1+\frac{t\left(r_{s}, g\right)^{2}}{\left|\varepsilon_{c}^{0}\right|}\right)} \\
\varepsilon_{c}^{G G A, p o l}\left(r_{s}, g\right)=\frac{\varepsilon_{c}^{1}}{1+\beta \ln \left(1+2^{-1 / 3} \frac{t\left(r_{s}, g\right)^{2}}{\left|\varepsilon_{c}^{1}\right|}\right)}
\end{array}\right.
$$

where $\beta=0,066725$ is determined so as to satisfy the slowly-varying limit, $t\left(r_{s}, g\right)=\frac{\pi^{4 / 3}}{3}\left(\frac{2}{3}\right)^{1 / 3} r_{s}^{7 / 2} g$ and $\varepsilon_{c}^{1}$ is analogous to $\varepsilon_{c}^{0}$ for the polarized uniform electron gas:

$$
\varepsilon_{c}^{1}=\varepsilon_{c}^{P W 91 L c}\left(r_{s}, \zeta=1\right)
$$

